\title{
Effect of climate change on horticultural crops
}

\begin{abstract}
The effect of global warming is now visible in many parts of the world. Abnormality in climate patterns, induced by accelerated warming, has started to affect a catchment-specific hydrologic cycle. Higher temperatures lead to a high rate of evaporation and dry conditions in some areas of the world. Severe weather events are now more common. Scientists believe that rapid warming in the last several decades is mostly due to human-induced changes in the atmosphere, on top of some natural variations. Impacts of climate change are complex as they can be both direct and indirect, the biggest casualty being natural resources such as agriculture. Agriculture is a carefully manipulated ecological system, the productivity of which could increase because higher levels of carbon dioxide in the atmosphere could allow a higher rate of photosynthesis. However, many interacting factors are at work. At higher levels of warming, estimated monetary impacts generally become negative, and studies, allowing for disastrous possibilities, can reach high negative outcomes. Moreover, the perennial plants (mostly fruit plants) are at more risk than annuals or seasonals.
\end{abstract}

Keyword: Climate change; Impact; Plant responses; Quality; Yield 